\title{
A comparative study of two tuning rules for delayed compensation controllers
}

\author{
Libor Pekařr,*, and Marek Kubalčík ${ }^{2}$ \\ ${ }^{1}$ Tomas Bata University in Zlín, Faculty of Applied Informatics, Department of Automation and Control Systems, Nad Stráněmi 4511, \\ 76005 Zlín, Czech Republic \\ ${ }^{2}$ Tomas Bata University in Zlín, Faculty of Applied Informatics, Department of Process Control, Nad Stráněmi 4511, 76005 Zlín, Czech \\ Republic
}

\begin{abstract}
The focus of this contribution is on the use of two controller tuning techniques for delayed controllers designed by an algebraic approach for linear time-invariant time delay systems. The well-known Chien-Hrones-Reswick (CHR) method and the Equalization Method (EM) are used. The tuning procedure is applied to compensation-type controllers that include internal delays, and hence there can be found a link to the habitual Smith predictor structure. This study considers two typical representatives of controlled plants with both input-output and internal delays; namely, the first- and second-order (of derivatives) systems are taken. Numerical comparative experiments are presented and discussed.
\end{abstract}

\section{Introduction}

It is common knowledge that majority of the controllers in the industry are linear covered by ProportionalIntegral (PI) or Proportional-Integral-Derivative (PID) ones. This is mainly because of their simplicity and robustness [1].

Due to the appearance and inclusion of delay effect in various technical as well as economic, social, biological, etc. systems, the family of time-delay systems (TDSs) have paid attention since the genesis of modern systems and control theory $[2,3]$.

A combination of PID control and TDSs plant, nevertheless, does not work well without extra effort of engineers when controller tuning since delay negatively influences dynamic properties of the feedback control system $[4,5]$. It is, for instance, possible to use a rational approximation of a TDS plant to get a finite-dimensional model [6,7], or optimization techniques (e.g., those minimizing integral criteria [8]). Time delay compensation techniques constitute another family of approaches to control TDSs. In 1957, Smith [9] investigated and published his famous deadtime compensation scheme, see Figure 1, in which $G_{0}(s)$ and $\tilde{G}_{0}(s)$ stand for the delay-free part of the plant $G$ and its model, respectively, and $C_{S}(s)$ expresses the particular controller. Last but not least, let us mention the finite spectrum assignment controller principle [10] that enables to prescribe and set an arbitrary finite number of feedback poles by using a convolution (i.e., a delay distribution) over state and/or input variables. Note that this feature cannot be satisfied via the use of PID or the Smith predictor.

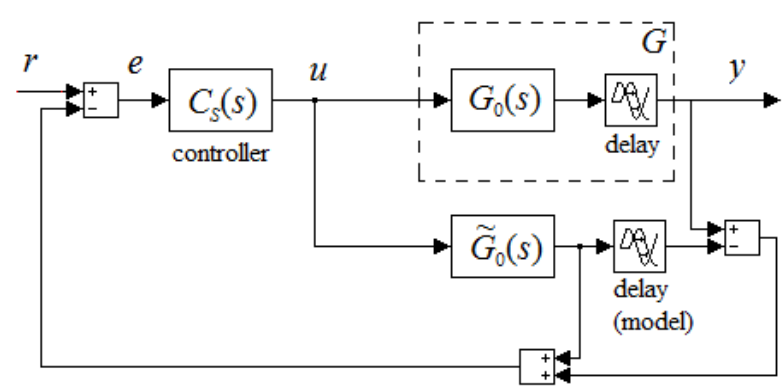

Fig. 1. Smith predictor.

By adopting a fractional system representation over a ring (without delay approximation), followed by an algebraic controller design with the solution of the linear Diophantine equation and subsequent Youla-Kučera controller parameterization $[11,12]$, an anisochronic (delayed) control law is obtained that can easily be compared to the Smith predictor for stable controlled plants [13]. In the delay-compensation controller structure, undefined parameters are to be suitably tuned.

The goal of this contribution is to provide the reader with a possible technique how to simply adopt some wellestablished tuning principles to the eventual controller structure, after some trivial asymptotic delay approximation. Its novelty lies in the combination of traditional rules and unconventional controllers; moreover, there is a lack of tuning ideas for such a class of controllers in the literature. Thus, the paper follows up on the above cited controller structure design published by the first author in the sense of a suitable setting of free controller parameter(s).

* Corresponding author: pekar@utb.cz 
We herein present two study cases; namely, the firstorder (of inertia) and the second order stable plants are considered. The simplified controller structures are of PI and PID types, respectively, on which the Equalization Method (EM) [14] and the Chien-Hrones-Reswick (CHR) tuning method [15] are applied, and results obtained by means of the Matlab/Simulink are compared via several performance measures.

The rest of the paper is organized as follows: The overview of used tuning techniques is given to the reader in section 2. Section 3 includes the derivation of particular tuning laws for two selected delayed plant models followed by numerical examples with simulations and qualitative evaluations. The paper is concluded in section 4.

\section{Tuning methods}

Since the controller structure design is not the primary aim of this study, we further do not deal with a presentation of the algebraic control design technique used to get non-quantified control laws, and hence, it is omitted. The reader is referred e.g. to $[12,13,16]$ for more detail.

Let us follow with particular PI and PID tuning rules herein used. Both the rules utilize the first order controlled plant model with input-output delay governed by the transfer function

$$
G(s)=\frac{K_{P} \exp (-\tilde{\tau} s)}{T s+1}
$$

where $K_{P}$ is the static gain, $T$ stands for the time constant, and $\tilde{\tau}$ means the modelled delay. The PID controller has the standard form as follows

$$
C(s)=K_{C}\left(1+\frac{1}{T_{I} s}+\frac{T_{D}}{T_{F} s+1}\right)
$$

where $K_{C}, T_{I}, T_{D}$ represent controller gain, integral and derivative time constants, respectively, and $T_{F} \gg T_{D}$ means the filter time constant.

\subsection{EM controller tuning rules}

For the PI rule (i.e., $T_{D}=0$ in (7)), the EM rules read [14]

$$
K_{C}=\frac{1+(1-\theta)^{2}}{2 K_{P}}, T_{I}=T_{a r} \frac{1+(1-\theta)^{2}}{2}
$$

where $T_{a r}=T+\tilde{\tau}$ is the average residence time, and $\theta=\tilde{\tau} / T_{a r}$.

The PID rule is expressed by the settings (4) where particular variables and symbols are further defined in (5).

$$
\begin{gathered}
K_{C}=\frac{1-\frac{2 \theta}{1+\sqrt{1+2 \theta^{2}}}}{K_{P}}, T_{I}=T_{a r} \frac{1+\sqrt{1+2 \theta^{2}}-2 \theta}{2}, \\
T_{D}=\frac{\left(T_{I}+T_{c r}\right) T_{c r}+\left(T_{I}-T_{a a}-T_{c a}\right) T_{a r}}{T} \\
T_{a r}=[\Gamma(s)]_{s=0}, T_{a a}=\frac{\left[-\Gamma^{\prime}(s)\right]_{s=0}}{T_{a r}}, \\
\Gamma(s)=\frac{1-G(s) / K_{P}}{s}, \Gamma^{\prime}(s)=\mathrm{d} \Gamma(s) / \mathrm{d} s, \\
T_{c r}=\tilde{\tau}\left(1-K_{P} K_{C}\left(1+\frac{\tilde{\tau}}{2 T}\right)\right), \\
T_{c a}=\frac{0.5 \tilde{\tau}^{2}}{T_{a r}}\left(1-K_{P} K_{C}\left(1+\frac{2 \tilde{\tau}}{3 T}\right)\right)
\end{gathered}
$$

\subsection{CHR controller tuning rules}

The PI tuning rules for the CHR method read

$$
K_{C}=\frac{0.35 T}{K_{P} \tilde{\tau}}, T_{I}=1.2 T
$$

and the PID rules are also expressed in simple forms as

$$
K_{C}=\frac{0.6 T}{K_{P} \tilde{\tau}}, T_{I}=T, T_{D}=0.5 \tilde{\tau}
$$

\section{Case studies}

\subsection{First order plant}

Let the controlled plant be governed by the transfer function

$$
G(s)=\frac{b \exp (-\tau s)}{s+a \exp (-\vartheta s)}
$$

where $\tau \geq 0$, and $0<a \vartheta<\pi / 2$ agrees with the stability condition.

The algebraic-based design for the simple negative feedback loop results in

$$
C(s)=\frac{\lambda}{b} \frac{s+a \exp \left(-\vartheta_{s}\right)}{s+\lambda(1-\exp (-\tau s))}
$$

where $\lambda>0$ represents a tuning parameter. By the comparison to the Smith predictor (see Figure 1), it is obtained that

$$
C_{S}(s)=\frac{\lambda}{b} \frac{s+a \exp \left(-\vartheta_{s}\right)}{s}
$$

In order to use tuning rules (3) and (6), let (9) be subject to the trivial approximation $\vartheta=\tau \rightarrow 0$, which yields 


$$
\widehat{C}(s)=\frac{\lambda}{b} \frac{s+a}{s}
$$

The controlled plant time constant $T$ can be taken in the sense of the first order system as $T=\left|s_{0}\right|^{-1}$ where $s_{0}$ is the dominant pole.

The equivalence of (3) and (11) for $K_{C}$ gives

$$
\lambda=a\left(\frac{1+(1-\theta)^{2}}{2}\right)
$$

while $T_{I}=a^{-1}$ is fixed due to (11).

In the contrary, the use of (6) results in

$$
\lambda=\frac{0.35 a T}{\tau}
$$

\subsubsection{Illustrative example}

Let the controlled plant be given by (8) with $a=0.2$, $b=0.6, \tau=4, \vartheta=0.8$. It can be found that $T=4.117$. From (12) and (13) one gets $\lambda=0.1257$ and $\lambda=7.205 \cdot 10^{-2}$, respectively. Particular control responses are displayed in Figure 2, where $r=\eta(t), d=\eta(t-75) \quad(\eta(t)$ means the Heaviside function, $r$ is the reference value and $d$ stands for the disturbance that acts on the control action). It is worth noting that $T_{I}=a^{-1}=5$ while (3) and (6) give, respectively, $T_{I}=4.9409$ and $T_{I}=5.1026$ - this proves that both the approaches are very close to the implicit result given by the algebraic approach.

Control performance (quality) measures that can judge control responses in a quantified way are given to the reader in Table 1. In the table, IAE $:=\int_{0}^{\infty}|e(t)| \mathrm{d} t, T_{95}$ expresses the duration until the output remains in the zone $\pm 5 \%$ around the reference, $\Delta e$ stands for the maximum relative overshoot while $r(t)=r$ is a constant and $d(t)=0$.

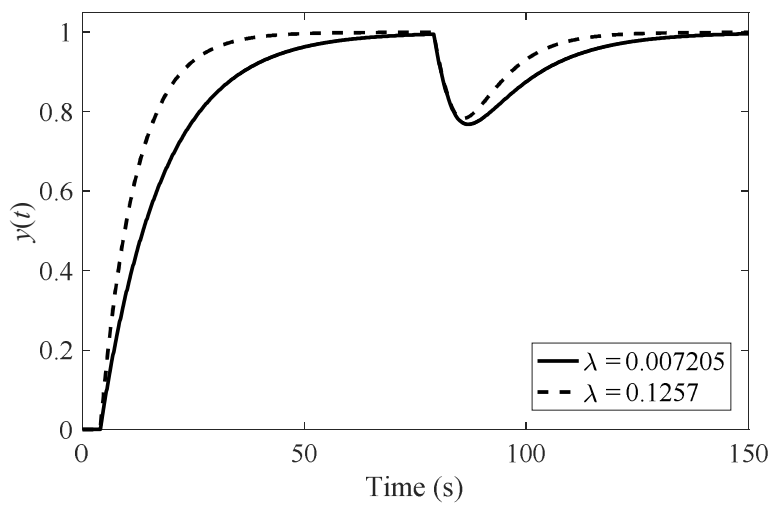

Fig. 2. Control responses comparison - the first order case.
Table 1. Control responses quality - the first order case.

\begin{tabular}{|c|c|c|c|}
\hline$\lambda$ & $\Delta e$ & $T_{95}$ & IAE \\
\hline 0.1257 & 0 & 27.8 & 12 \\
\hline $7.205 \cdot 10^{-2}$ & 0 & 45.6 & 17.9 \\
\hline
\end{tabular}

It can be clearly deduct that the EM gives better results (in terms of the selected performance measures). Note, however, that neither the disturbance nor the control action is taken into account here.

\subsection{Second order plant}

Assume the plant (model) transfer function as

$$
G(s)=\frac{b \exp (-\tau s)}{\left(s+a_{1} \exp (-\vartheta s)\right)\left(s+a_{2}\right)}
$$

with $\tau \geq 0,0<a_{1} \vartheta<\pi / 2, a_{2}>0$.

The coprime factorization, as a part of the algebraic control design procedure, admits several options, which yields different eventual control laws. Consider two of them as follows:

$$
C(s)=\frac{\eta}{b} \frac{\left(s+a_{1} \exp \left(-\vartheta_{s}\right)\right)\left(s+a_{2}\right)}{s^{2}+\gamma s+\eta(1-\exp (-\tau s))}
$$

where

$$
\eta=\lambda^{2}, \gamma=2 \lambda
$$

or

$$
\eta=\lambda_{1} \lambda_{2}, \gamma=\lambda_{1}+\lambda_{2}
$$

for some $\lambda, \lambda_{1}, \lambda_{2}>0$. Under the simplification $\vartheta=\tau \rightarrow 0$, one gets

$$
\widehat{C}(s)=\frac{\eta}{b} \frac{\left(s+a_{1}\right)\left(s+a_{2}\right)}{s(s+\gamma)}
$$

Nevertheless, whenever (4) and (5), or (7) are applied to (18), it is not possible to solve this task analytically; hence, a numerical computation ought to be used. Notice that there are one or two free parameters to be obtained by the solution of three equations. Note, moreover, that the plant time constant and delay can be estimated e.g. by using the following rules

$$
T=\left|s_{0}\right|^{-1}, \tilde{\tau}=\tau+\left|s_{1}\right|^{-1}
$$

see [17], where $s_{1}$ stands for the dominant pole of the spectrum excluding $s_{0}$. 


\subsubsection{Illustrative example}

Consider the controlled plant (14) with the following parameters: $\quad a_{1}=0.2, \quad a_{2}=0.5, \quad b=0.6, \quad \tau=4$, $\vartheta=0.8$.

As first, assume the EM and option (16). By applying (5), (6), such that conditions for $K_{C}, T_{I}, T_{D}$ are satisfied, one gets, respectively

$$
\lambda=0.2526, \lambda=7.3324, \lambda=0.0882
$$

Now, consider the second option (17). Then, by matching this formula with pairs $\left\{K_{C}, T_{I}\right\},\left\{K_{C}, T_{D}\right\}$ from (5), (6), it is, respectively, obtained

$$
\begin{aligned}
& \lambda_{1}=0.092, \lambda_{2}=14.57, \\
& \lambda_{1,2}=0.088 \pm 0.276 \mathrm{j}
\end{aligned}
$$

The remaining pair $\left\{T_{I}, T_{D}\right\}$ cannot be used doe to algebraic (solvability) issues.

Regarding the CHR method, the following result is obtained by matching (7) and (16) for the same set of controller variables as introduced above (20):

$$
\lambda=4.3061, \lambda=0.1734, \lambda=0.0851
$$

Finally, the CHR method given by (7) applied to (17) gives

$$
\begin{aligned}
& \lambda_{1,2}=0.173 \pm 1.104 \mathrm{j}, \\
& \lambda_{1,2}=0.085 \pm 1.149 \mathrm{j}
\end{aligned}
$$

for pairs $\left\{K_{C}, T_{I}\right\},\left\{K_{C}, T_{D}\right\}$, respectively.

Due to a wide range of values, only some distinct values from (20)-(23) have been eventually taken, and the corresponding control responses are displayed in Figure 3. Note that reference and disturbance signals are identical with those in the preceding example.

The corresponding control performance measures are given to the reader in Table 2 .

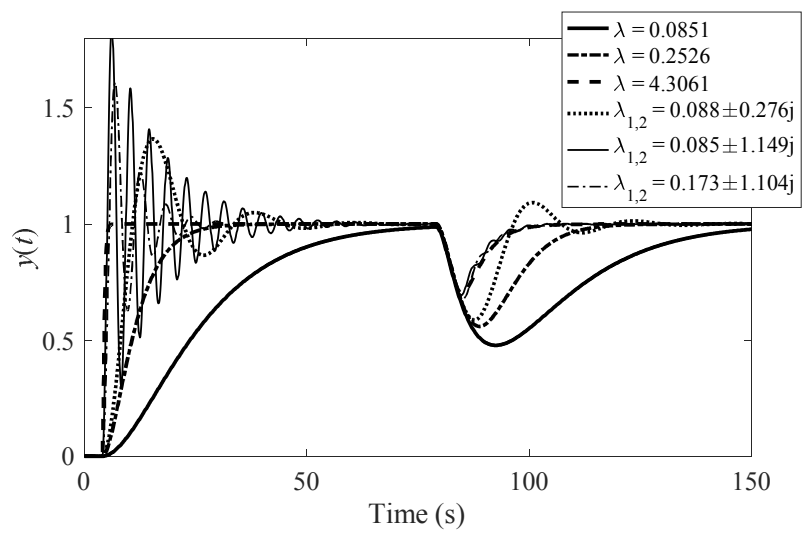

Fig. 3. Control responses comparison - the second order case.
Table 2. Control responses quality - the second order case.

\begin{tabular}{|c|c|c|c|}
\hline$\lambda, \lambda_{1}, \lambda_{2}$ & $\Delta e$ & $T_{95}$ & IAE \\
\hline 0.2526 & 0 & 22.8 & 12 \\
\hline $0.088 \pm 0.276 \mathrm{j}$ & 0.367 & 31.5 & 12.1 \\
\hline 4.3061 & $3.8 \cdot 10^{-5}$ & 17.9 & 4.5 \\
\hline 0.0851 & 0 & 59.7 & 27.3 \\
\hline $0.173 \pm 1.104 \mathrm{j}$ & 0.61 & 21.3 & 7.8 \\
\hline $0.085 \pm 1.149 \mathrm{j}$ & 0.836 & 17.6 & 11.5 \\
\hline
\end{tabular}

It seems that a higher value of $\lambda$ yields a better control response. However, it is sacrificed to significant abrupt changes in the control action (not displayed herein). In the contrary, low values of $\lambda$ give very slow responses. Regarding complex conjugate pairs $\lambda_{1,2}$, it is apparent that a higher ratio of the particular imaginary and real parts results in a higher amplitude of oscillations; however, the settling time is reduced. The higher is the real part of the pair $\lambda_{1,2}$, the lower the integral error is.

\section{Conclusions}

In this contribution, a brief comparative study on the application of two well-established controller tuning methods to algebraic-based controllers has been presented. The controller has had a compensation (or, predictor) structure to attenuate the influence of input-output or internal (state) delays; however, its design has not been presented here - the reader has been referred to particular literature resources. As the tuning techniques, the Equalization Method and the Chien-Hrones-Reswick method have been applied to a trivial finite-dimensional approximation of the delayed controller. The application of well-established tuning rules to this class of controllers has been the main contribution of the paper. Two types of controlled plants have been considered; namely, stable systems of the first and second order. Numerical tests have indicated that the Equalization Method have provided better results in both the cases, and, surprisingly, a single tuning parameter has been a better choice compared to two degrees of freedom in the latter case.

A possible further research direction in this field can be dedicated to the extension of presented principles to unstable systems, or to a thorough comparative study on the efficiency and performance of various wellestablished tuning rules.

This work was performed with the financial support by the Ministry of Education, Youth and Sports of the Czech Republic within the National Sustainability Programme project No. LO1303 (MSMT-7778/2014).

\section{References}

1. K.J. Aström, T. Hägglund, Advanced PID control (ISA, Research Triangle Park, 2006)

2. J.-P. Richard, Automatica 39, 1667-1694 (2003) 
3. E. Fridman, Introduction to time-delay systems: Analysis and control (Birkhäuser, Basel, 2014)

4. M. Huba, IFAC Proc. Vol. 36, 53-58 (2003)

5. Y. Xia, M. Fu, P. Shi, Analysis and synthesis of dynamical systems with time-delays (SpringerVerlag, Berlin, 2009)

6. R. Prokop, J.-P. Corriou, Int. J. Control 66, 905-921 (1997)

7. L. Pekař, E. Kurečková, Int. J. Math. Comput. Simul. 6, 153-160 (2012)

8. P. Zítek, J. Fišer, T. Vyhlídal, IFAC-PapersOnLine, 49, 177-181 (2016)

9. O.J.M. Smith, Chem. Eng. Prog. 53, 217-219 (1957)

10. A.V. Metel'skii, Differ. Equ. 49, 1405-1422 (2013)

11. V. Kučera, Automatica 29, 1361-1375 (1993)

12. L. Pekař, R. Prokop, B. Pol. Acad. Sci-Tech. 65, 341-350 (2017)

13. R. Prokop, L. Pekař, Automatizace 52, 414-418 (2009)

14. R. Gorez, P. Klán, IFAC Proc. Vol. 33, 133-140 (2000)

15. K.L. Chien, J.A. Hrones, J.B. Reswick, Trans. Am. Soc. Mech. Eng. 74, 175-185 (1952)

16. L. Pekař, P. Valenta, Int. J. Math. Mod. Appl. Sci. 7, 311-319 (2013)

17. M. Vítečková, A. Víteček, in Proc. 12th Carpat. Control Conf. 2011, 429 - 432 (2011) 